\title{
Electrospun Borneol-PVP Nanocomposites
}

\author{
Xiao-Yan Li, Xia Wang, Deng-Guang Yu, Shuai Ye, Qi-Kun Kuang, \\ Qing-Wen Yi, and Xin-Zhe Yao
}

School of Materials Science and Engineering, University of Shanghai for Science and Technology, Shanghai 200093, China

Correspondence should be addressed to Xiao-Yan Li, lixiaoyan@usst.edu.cn and Deng-Guang Yu, ydg017@usst.edu.cn

Received 13 January 2012; Accepted 5 March 2012

Academic Editor: Haifeng Chen

Copyright ( $) 2012$ Xiao-Yan Li et al. This is an open access article distributed under the Creative Commons Attribution License, which permits unrestricted use, distribution, and reproduction in any medium, provided the original work is properly cited.

The present work investigates the validity of electrospun borneol-polyvinylpyrrolidone (PVP) nanocomposites in enhancing drug dissolution rates and improving drug physical stability. Based on hydrogen bonding interactions and via an electrospinning process, borneol and PVP can form stable nanofiber-based composites. FESEM observations demonstrate that composite nanofibers with uniform structure could be generated with a high content of borneol up to $33.3 \%(\mathrm{w} / \mathrm{w})$. Borneol is well distributed in the PVP matrix molecularly to form the amorphous composites, as verified by DSC and XRD results. The composites can both enhance the dissolution profiles of borneol and increase its physical stability against sublimation for long-time storage by immobilization of borneol molecules with PVP. The incorporation of borneol in the PVP matrix weakens the tensile properties of nanofibers, and the mechanism is discussed. Electrospun nanocomposites can be alternative candidates for developing novel nano-drug delivery systems with high performance.

\section{Introduction}

Electrospinning is a popular procedure in scientific researches due to the ease of implementation, the cost-effectiveness of the process, and the unique properties and potential applications of the resultant nanofibers [1-4]. It has a strong capability of producing nanofibers of polymers and some small molecules such as phospholipids and cyclodextrin, of giving the nanofibers with secondary structural characteristics by copying the spinnerets such as coaxial and side-by-side, and of generating polymer-based composites through the interactions between the filament-forming polymer matrix and the functional ingredients [5-9].

Nanocomposites have drawn considerable attentions because of the ability to produce high-performance materials with enhanced or novel properties $[10,11]$. As a simple one-step top-down process for preparing one-dimensional (1D) nanofibers, electrospinning exhibits great capability in preparing nanoscale polymer composites owing to the tremendous rapid drying process, often in a time scale of $10^{-2} \mathrm{~s}$ [12-16]. It has been demonstrated that electrospun nanofiber composites can enhance the functions of active ingredients and even achieve new functions by taking advantages of their unique properties, such as continuous three-dimensional web structure, thin diameter, large surface area, and high porosity [17-19].

Borneol, a compound derived from ryobalanops aromatica (a tree that belongs to the teak family), is a common ingredient in many traditional Chinese herbal formulas. Borneol has a wide range of uses. It aids the digestive system by stimulating the production of gastric juices; tones the heart and improves circulation, treats bronchitis, coughs and colds, can relieve pain caused by rheumatic diseases and sprains, reduces swelling, relives stress, and can be used as a tonic to promote relaxation and reduce exhaustion. In some parts of the world, it is even used as an insect repellant. However, borneol has very poor water solubility, poor physical stability due to easy sublimation, and side effect of resulting in irritation [20-22].

Based on above-mentioned knowledge, we presently investigate that electrospinning is exploited to prepare composites of borneol and hydrophilic excipients in the form of nanofibers. Polyvinylpyrrolidone was selected as the filament-forming matrix because it has been broadly reported to 


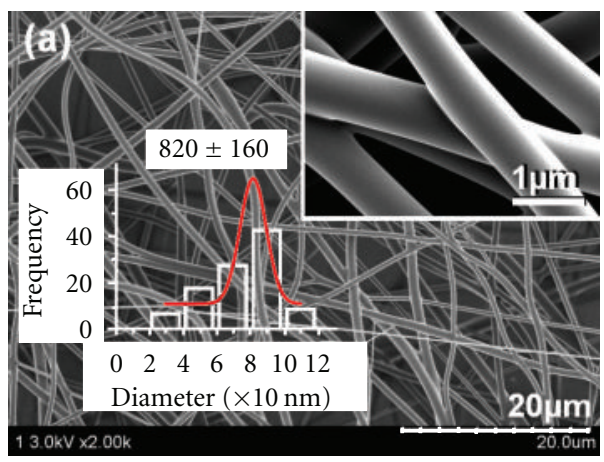

(a)

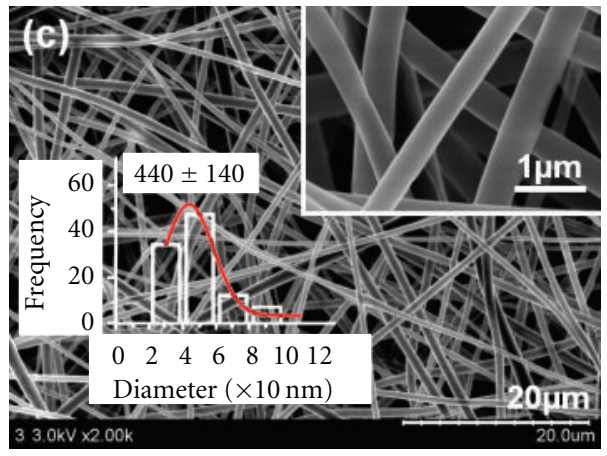

(c)

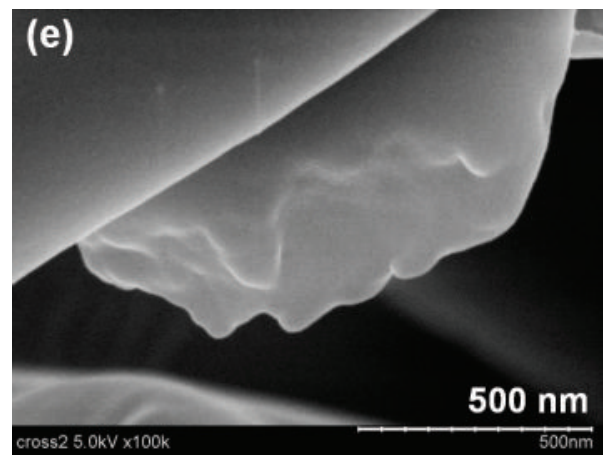

(e)

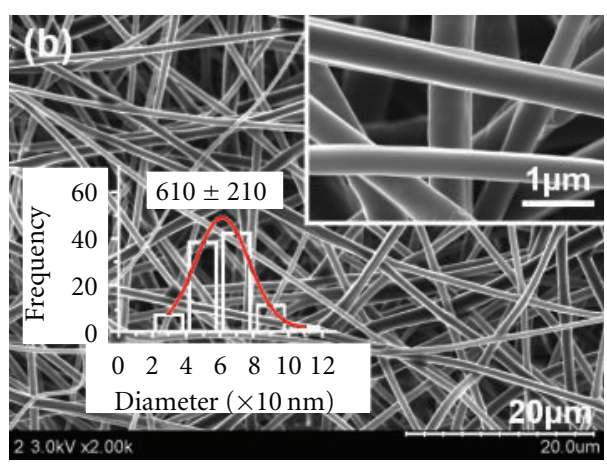

(b)

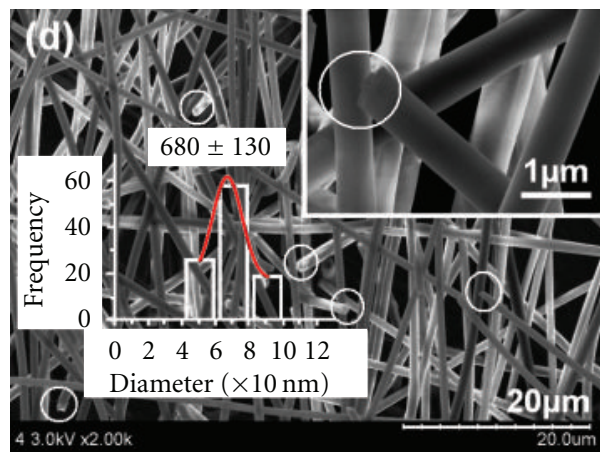

(d)

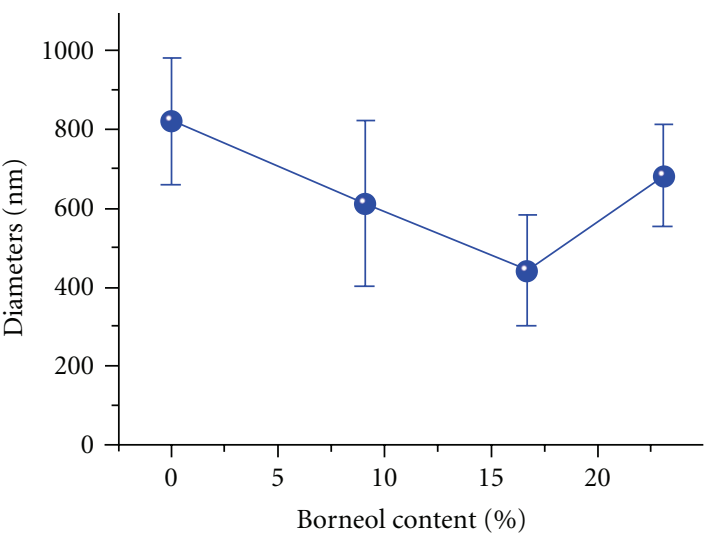

(f)

FIGURE 1: FESEM images: (a)-(d) surface morphology of the composite nanofibers F0, F1, F2, and F3, respectively; (e) cross-section of the composite nanofibers F3; (f) relationship between fiber diameter and borneol content.

be used for enhancing solubility of a wide variety of poorly water-soluble drug and it has good electrospinnability in a series of typical organic solvents such as methanol, ethanol, chloroform, and N, N-dimethylacetamide [23-26].

\section{Experimental}

2.1. Materials. Borneol (purity over 95\%) was purchased from Shanghai Winherb Medical S \& T Development Co., Ltd (Shanghai, China). Polyvinylpyrrolidone K60 (PVP K60, $M=360,000)$ was obtained from the Shanghai Yunhong Pharmaceutical Aids and Technology Co., Ltd. (Shanghai,
China). Anhydrous ethanol was provided by the Sinopharm Chemical Reagent Co., Ltd. All other chemicals used were analytical grade, and ultra-high-quality water was used.

\subsection{Preparation}

Preparation of Spinning Solutions. Borneol and the polymer PVP K60 were dissolved in ethanol in turn at the ambient temperature $21^{\circ} \mathrm{C}$. The concentration of PVP was fixed at 10 $(\mathrm{w} / \mathrm{v}) \%$. Four types of spinning solution with varied concentrations of borneol 0, $1(\mathrm{w} / \mathrm{v}) \%, 2(\mathrm{w} / \mathrm{v}) \%$, and $5(\mathrm{w} / \mathrm{v}) \%$ (Table 1) were prepared, and the composite nanofiber mats 


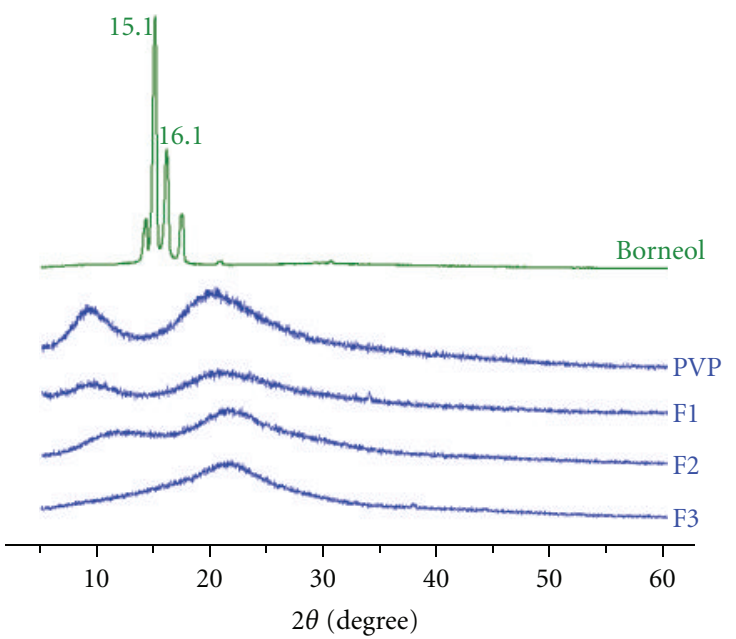

Figure 2: X-ray diffraction patterns of the components (borneol and PVP) and their nanofibers with different ratio of borneol to PVP.

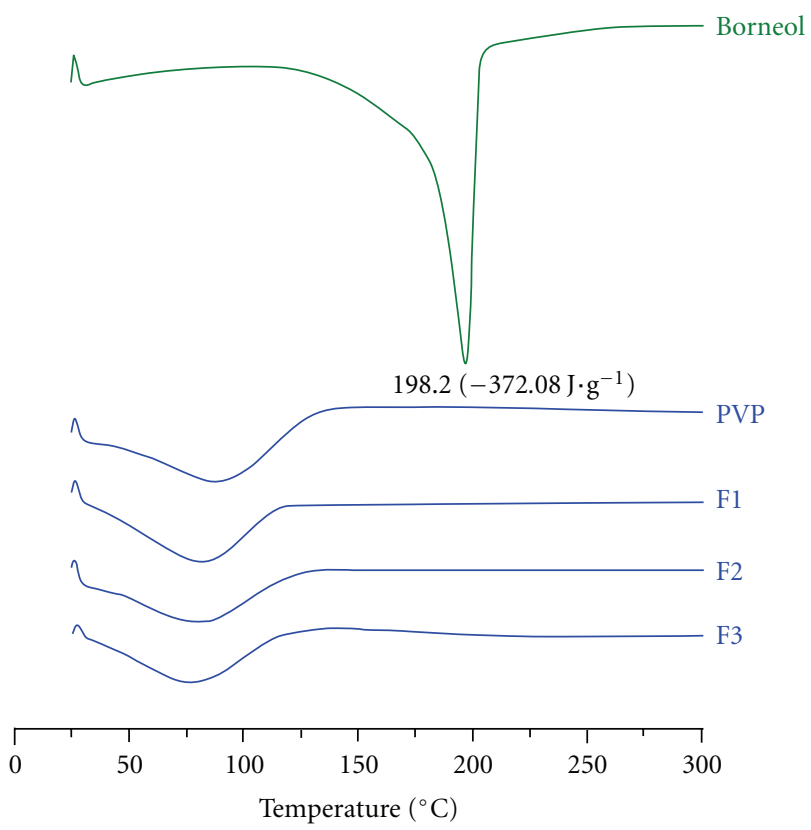

FIGURE 3: DSC thermograms of the components (borneol and PVP) and their nanofibers with different ratio of borneol to PVP.

were denoted as F0, F1, F2, and F3, respectively. Mechanical stirring was applied for $1 \mathrm{~h}$ to obtain homogeneous codissolved spinning solutions. The solutions were degassed with a SK5200H ultrasonator (350W, Shanghai Jinghong Instrument Co., Ltd. Shanghai, China) for 10 minutes before electrospinning.

Electrospinning Process. A high-voltage power supply (Shanghai Sute Electrical CO., Ltd., Shanghai, China) was used to provide high voltages in the range of $0-60 \mathrm{kV}$. To avoid carrying any air bubbles, spinning solutions were carefully loaded in a $10 \mathrm{~mL}$ syringe to which a stainless steel

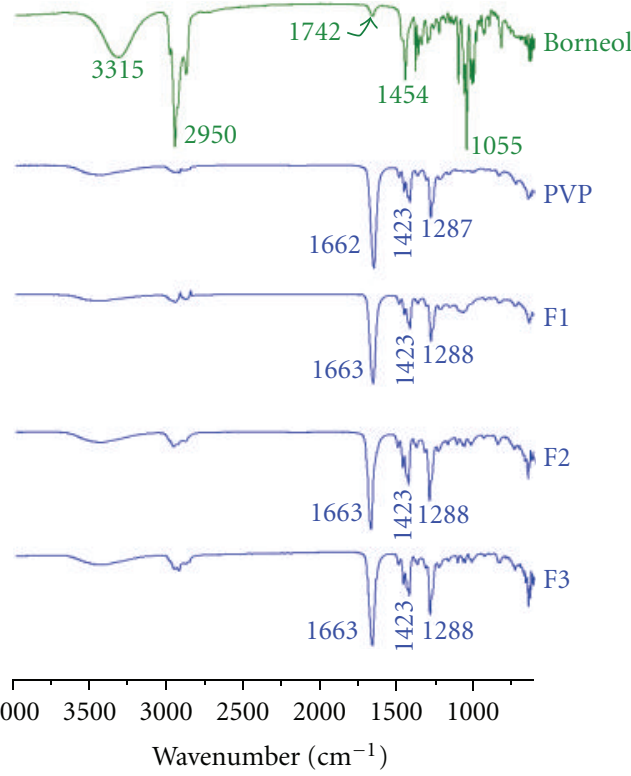

FIGURE 4: ATR-FTIR spectra of the components (borneol and PVP) and their nanofibers with different ratio of borneol to PVP.

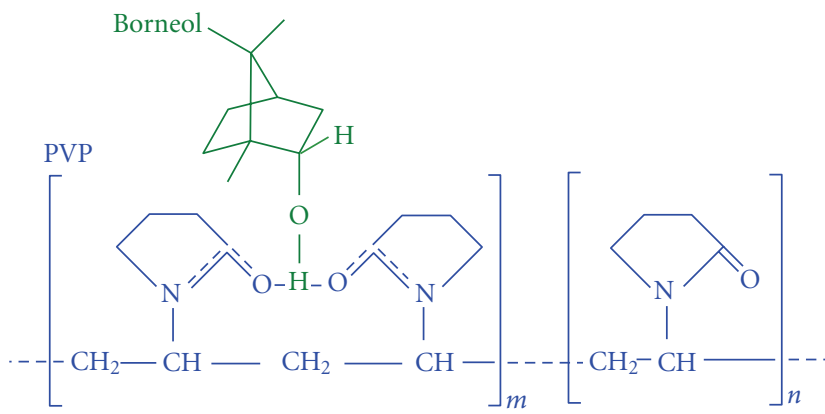

Figure 5: Molecular structures of the components (borneol and PVP) and the hydrogen bonding between them.

capillary metal-hub needle was attached. The inside diameter of the metal needle was $0.5 \mathrm{~mm}$. The positive electrode of the high-voltage power supply was connected to the needle tip and the grounded electrode was linked to a metal collector wrapped with aluminum foil.

The electrospinning process was carried out under ambient conditions $\left(21 \pm 2^{\circ} \mathrm{C}\right.$ and relative humidity $61 \pm$ $3 \%$ ). A fixed electrical potential of $12 \mathrm{kV}$ was applied across a fixed distance of $15 \mathrm{~cm}$ between the tip, and the collector. The feed rate of solutions was controlled at $2.0 \mathrm{~mL} \cdot \mathrm{h}^{-1}$ by means of a single syringe pump (KDS100, Cole-Parmer, Vernon Hills, IL, USA).

2.3. Characterization. The morphology of the surface, crosssections of the nanofiber mats, and the casting films were assessed using a S-4800 field emission scanning electron microscope (FESEM) (Hitachi, Japan). The average fiber diameter was determined by measuring diameters of fibers at over 100 points from FESEM images using NIH Image J software (National Institutes of Health). 

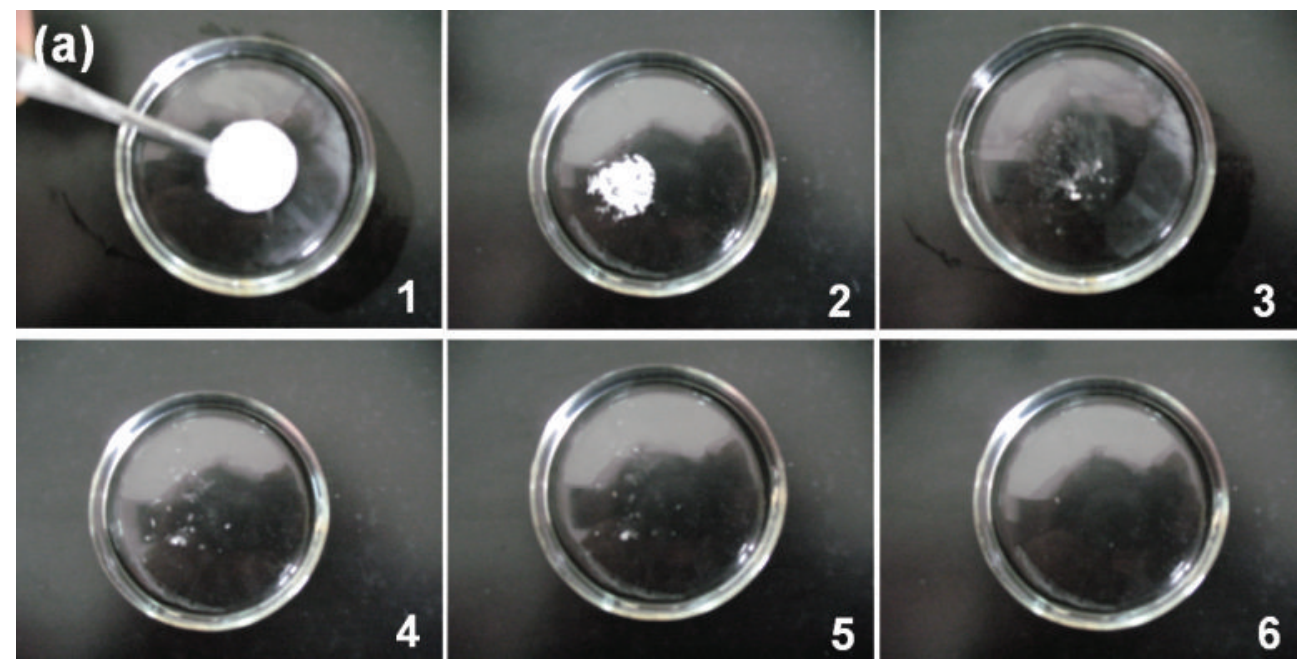

(a)

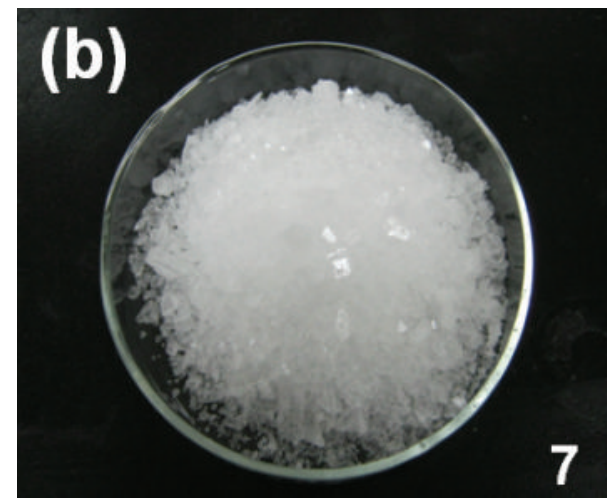

(b)

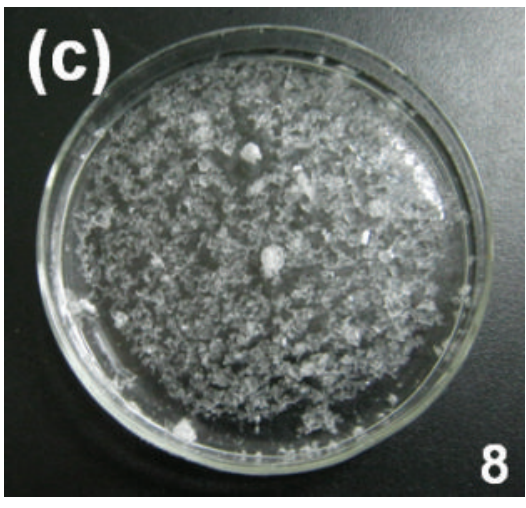

(c)

FIGURE 6: Dissolving tests: (a) photographs of the disintegrating process of F3, the fast-dissolving process of F3 is shown in sequence from 1 to 6; (b) pure borneol particle; (c) the dissolving status of borneol.

TABLE 1: Preparation conditions for the borneol-PVP nanocomposites.

\begin{tabular}{lcccc}
\hline & F0 & F1 & F2 & F3 \\
\hline$C_{\mathrm{PVP}}{ }^{\mathrm{a}}(\mathrm{w} / \mathrm{v} \%)$ & & 10 & & \\
$\mathrm{C}_{\mathrm{b}}{ }^{\mathrm{b}}(\mathrm{w} / \mathrm{v} \%)$ & 0 & 1 & 2 & 5 \\
$\mathrm{P}_{\mathrm{b}}{ }^{\mathrm{c}}(\mathrm{w} / \mathrm{w} \%)$ & 0 & 9.1 & 16.7 & 33.3 \\
\hline
\end{tabular}

${ }^{a}$ CPVP: concentration of PVP in spinning solutions.

${ }^{\mathrm{b}} \mathrm{C}_{\mathrm{b}}$ : concentration of borneol in spinning solutions.

${ }^{c} \mathrm{P}_{\mathrm{b}}$ : percentage borneol in the nanofibers, $\mathrm{P}_{\mathrm{b}}=\mathrm{C}_{\mathrm{b}} /\left(\mathrm{C}_{\mathrm{PVP}}+\mathrm{C}_{\mathrm{b}}\right) \times 100 \%$.

The differential scanning calorimetry (DSC) analyses were carried out using an MDSC 2910 differential scanning calorimeter (TA Instruments Co., USA). Sealed samples were heated at $10^{\circ} \mathrm{C} \cdot \mathrm{min}^{-1}$ from 21 to $300^{\circ} \mathrm{C}$. The nitrogen gas flow rate was $40 \mathrm{~mL} \cdot \mathrm{min}^{-1}$.

X-ray diffraction analyses (XRD) were obtained on a D/Max-BR diffractometer (Rigaku, Japan) with $\mathrm{Cu} \mathrm{K} \alpha$ radiation in the $2 \theta$ range of $5-60^{\circ}$ at $40 \mathrm{mV}$ and $300 \mathrm{~mA}$.

Attenuated total reflectance Fourier transform infrared (ATR-FTIR) analysis was carried out on a Nicolet-Nexus 670
FTIR spectrometer (Nicolet Instrument Corporation, Madison, USA) over the range $500-4000 \mathrm{~cm}^{-1}$ and a resolution of $2 \mathrm{~cm}^{-1}$.

2.4. Properties of the Borneol-PVP Nanofiber Mats. To determine the effect of composites in improving borneol physical stability, a comparison study was conducted. $50 \mathrm{mg}$ of borneol particles (pulverized to pass through a $125 \mu \mathrm{m}$ mesh sieve) fiber mats containing $50 \mathrm{mg}$ borneol (i.e., $550 \mathrm{mg}$ fibers F1, $300 \mathrm{mg}$ fibers F2 and $150 \mathrm{mg}$ fibers F3) were placed on the petri dishes in the atmosphere. Their weights were measured every $24 \mathrm{~h}$.

A petri dish of water was used to demonstrate the speed of the dissolving process of the borneol-PVP nanofiber mats. The dissolving processes were recorded at 30 frames per second with a digital video recorder (Canon PowerShot A490, Tokyo, Japan).

Tensile tests were measured according to ASTM D882 using a universal testing machine (Instron5566, Instron, Canton, MA). All samples were cut to the standard dumbbell shape, conditioned overnight $\left(21 \pm 1^{\circ} \mathrm{C}\right.$, relative humidity of $65 \pm 2 \%$ ) before testing. 


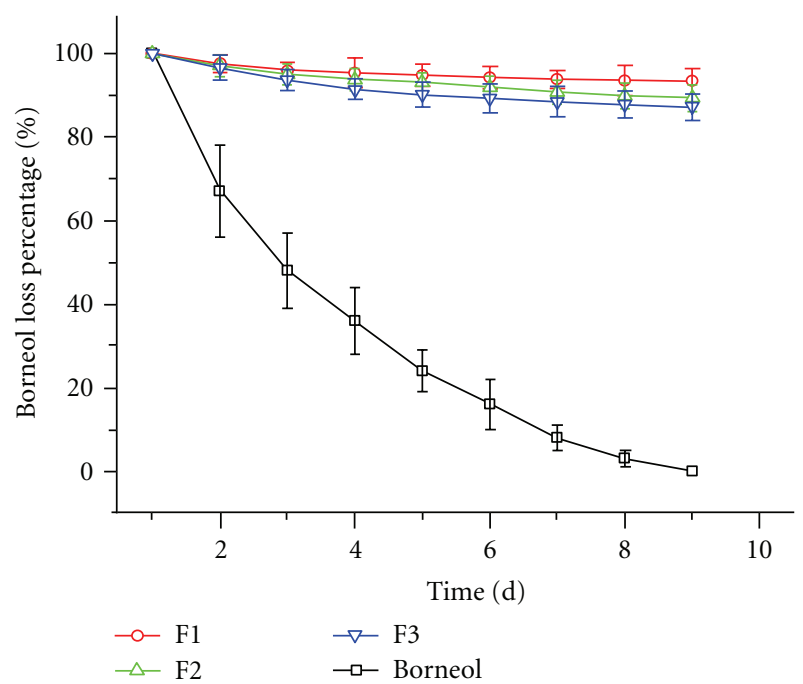

FIGURE 7: Weight loss of pure borneol and their nanofibers with different ratio of borneol to PVP in the atmosphere.

\section{Results and Discussion}

3.1. Morphology. Figures $1(\mathrm{a})-1(\mathrm{~d})$ show FESEM images of PVP nanofibers with a content of borneol at $0 \%$ (F0), 9.1\% (F1), 16.7\% (F2), and 33.3\% (F3) (w/v), respectively. All the four types of nanofibers have uniform structures without beads-on-a-string morphology, they had smooth surfaces, and the matrix was free of any separating particles. However, the fibers F3 were very crisp, as verified by circle in Figure $1(d)$ in which the cross-sections of nanofibers should be generated during the sampling process.

Figure 1(e) shows FESEM images of the cross-section of the composite nanofibers F3. It is clear that there are no apparent particles within them, indicating that no phase separating had occurred during electrospinning. This also suggests that borneol molecules are uniformly distributed throughout the PVP matrix.

As the concentrations of borneol increased, the average diameters of the nanofibers gradually decreased (F0 to F1 and F2). The addition of borneol in PVP solutions can increase their conductivities and thereby enhanced the electrical drawing effects on the jet fluids, resulting thinner nanofibers [12]. However, when the drug loading was further increased, the average diameters of nanofibers F3 increased (F3). As the concentration of borneol further increased, the solution viscosity also increased, and this counteracted the influence of conductivity increases and gradually had a greater influence on nanofiber diameter compared to electrical forces [27]. The relationship between fiber diameter and borneol content is shown in Figure 1(f).

\subsection{Physical Status of the Components in the Electrospun} Fibers. DSC and XRD tests were undertaken to determine the physical status of borneol in the composite nanofibers. As shown in Figure 2, the presence of distinct peaks in the XRD patterns indicated that borneol was present as crystalline materials with characteristic diffraction peaks at

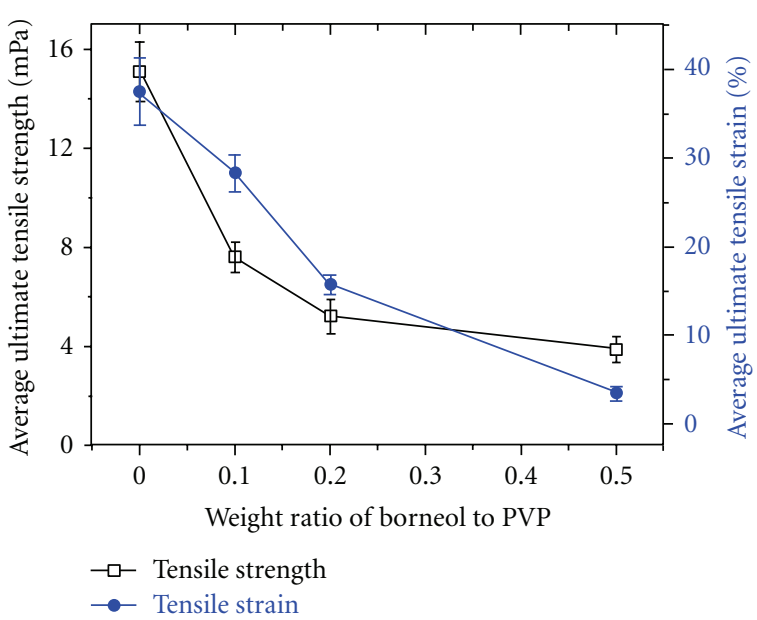

Figure 8: Tensile properties of electrospun borneol-PVP nanocomposite fibers with different ratio of borneol to PVP.

$15.1^{\circ}$ and $16.1^{\circ}$. The PVP diffraction exhibits a diffused background pattern with two diffraction halos indicating that the polymer is amorphous. In respect to the composite nanofibers F1 to F3, the characteristic peaks of borneol were absent. This suggests that borneol were no longer present as crystalline material, but was converted into an amorphous state.

The DSC thermograms are shown in Figure 3, and the DSC curve of pure borneol exhibited a single endothermic response corresponding to melting points of $198.2^{\circ} \mathrm{C}$ $\left(-372.08 \mathrm{~J} \cdot \mathrm{g}^{-1}\right)$. As an amorphous polymer PVP K60 did not show any fusion peaks or phase transitions, apart from a broad endotherm, this being due to dehydration, which lies between 80 and $120^{\circ} \mathrm{C}$ and with a peak at $85^{\circ} \mathrm{C}$. DSC thermograms of the composite nanofibers did not show any melting peaks of borneol. All the borneol-PVP composite nanofibers had a broad endotherm ranging from about 60 to $100^{\circ} \mathrm{C}$. The results form DSC and XRD similarly demonstrated that borneol was no longer present as a crystalline material but had been converted into amorphous composites with matrix PVP.

3.3. The Secondary Interactions among the Components. The compatibility among the components is essential for producing high quality and the stability of the composite nanofibers. Often the second-order interactions such as hydrogen bonding, electrostatic interactions, and hydrophobic interactions would improve their compatibility [12]. Each borneol molecule has one $-\mathrm{OH}$ group whereas PVP molecules have numerous $-\mathrm{C}=\mathrm{O}$ groups. So they can form composites with borneol acting as proton donors and PVP molecules acting as proton receptors.

This can be as verified by the ATR-FTIR spectra in which sharp peaks were visible for pure crystalline borneol at 2950, 1454, and $1055 \mathrm{~cm}^{-1}$ (Figure 4). However, all the peaks for borneol disappeared in the composite nanofibers when samples were run in the ATR-FTIR spectra, jointly demonstrating that hydrogen bonding occurred (Figure 5). 


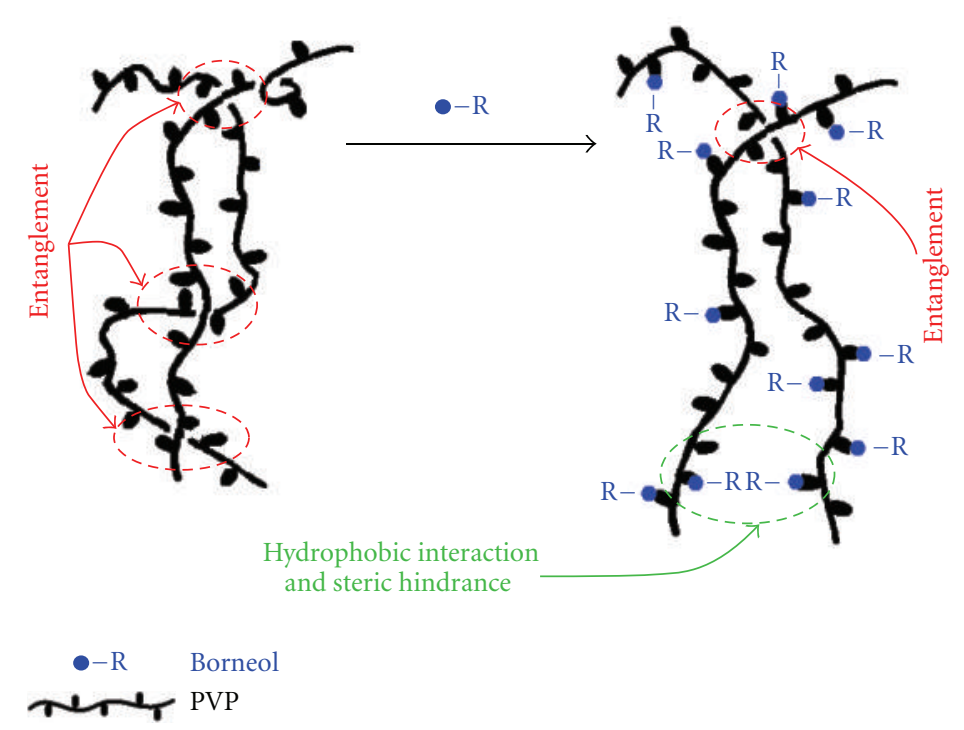

FIgURE 9: Influence of borneol on the properties of PVP matrix nanofibers.

By the way, there is a tiny peak of $1742 \mathrm{~cm}^{-1}$ in spectra of borneol in Figure 4, reflecting that some borneol had been oxidized to camphor.

Electrospinning is not only a useful tool for fabricating 1D polymer nanofibers, but also an excellent process for generating composites with the functional gradients dispersing on the filament-forming polymer matrix molecularly $[7,28]$. Here, PVP can provide functional groups for "anchoring" borneol to amorphize them during the fast drying electrospinning process. Only the rapid evaporation of the solvent and the favorable interactions between the ingredients that resulted in the nanofiber composites, in which borneol molecules were distributed through the PVP matrix with their physical state in the liquid solutions to be propagated into the solid nanofibers and lose the original crystal state as pure materials.

Discs with a diameter of $16 \mathrm{~cm}$ were cut from the composite nanofiber mats F0 to F3 for fast dissolving tests. Pure borneol particles were used as controls. Shown in Figure 6(a) is a typical disintegrating process of nanofiber F3, and Figures 6(b) and 6(c) show the borneol particles and their insolubility in water. The dissolution of F3 cost $14.5 \pm 2.4 \mathrm{~s}(n=6)$. The average disappearance times for nanofiber mats F0 to F2 in water were $13.8 \pm 1.2 \mathrm{~s}, 14.3 \pm$ $2.2 \mathrm{~s}$, and $14.3 \pm 2.6 \mathrm{~s}$, respectively. The disintegrating times were not significantly different statistically $(P>0.05$, paired $t$-test) between the pure PVP nanofibers and the borneol-contained nanofibers, suggesting that the poorly water-soluble borneol can be "led" to quickly dissolve by hydrophilic PVP molecules through their composites. The $3 \mathrm{D}$ continuous web structure, the big surface resulting from the small diameter of nanofibers, and the amorphous status of borneol in the PVP matrix synergistically acted to promote the fast dissolution of borneol.

Shown in Figure 7 is the weight loss of the borneol particles and its electrospun composites. The $50 \mathrm{mg}$ borneol particle totally disappeared in the ninth day, whereas all the composites still immobilized over $92 \%$ of the contained borneol. The hydrogen bonding between PVP and borneol effectively kept the drug from sublimation from the composites, demonstrating that the nanofiber mats could greatly increase borneol physical stability.

The ultimate tensile strength and tensile strain for all the electrospun borneol/PVP nanofibrous membranes with various borneol contents were tested and summarized (Figure 8). This process characterises the relationship between the average ultimate tensile strength, average ultimate tensile strain, and borneol content. The neat PVP nanofibers showed highest tensile strain. Increasing the weight ratio of borneol showed a decrease in the average tensile strength.

PVP is a linear polymer and has good electrospinnability. When borneol is added to PVP solutions, it is able to interact with PVP molecules through hydrogen bonding. The PVP and borneol molecules together are likely to behave like branched polymers the hydrophobic interactions and stereic hindrance resulting from the branched-borneol would change the nanofibers' matrix PVP chain conformation and PVP-PVP molecular interactions and so decreasing their direct entanglements (Figure 9).

Thus, the presence of borneol affects the stiffness of the PVP main chains and distributes the stretching properties in the chains of PVP, and the disturbance was more significant with the higher addition percentage of borneol, resulting in the obvious decrease of the average tensile strength and strain of borneol-PVP composite nanofiber mats. These results confirm observation obtained from FESEM and also the results of FT-IR studies.

\section{Conclusions}

With hydrophilic polymer PVP as the filament-firming matrix, several borneol-PVP nanocomposites with varied drug content have been successfully prepared using a single 
fluid electrospinning process. FESEM observations demonstrate that composite nanofibers with uniform structure could be generated with a high content of borneol up to $33.3 \%(w / w)$. Borneol was well distributed in the PVP matrix molecularly to form the amorphous composites, as verified by DSC and XRD results. The composites effectively enhanced the dissolution profiles of borneol within a time period of $15 \mathrm{~s}$ and they were also able to increase borneol physical stability against sublimation for long-time storage by immobilization of borneol molecules with PVP through hydrogen bonding. The incorporation of borneol in the PVP matrix weakens the tensile properties of nanofibers. The present strategy shows an avenue that a wide variety of volatile functional active pharmaceutical ingredients (no matter liquid or solid substance) can be immobilized using electrospun nanofibers for easy development of novel nanodosage form.

\section{Acknowledgments}

This work was supported by the scientific starting funds for young teachers of the University of Shanghai for Science and Technology (no. 10-00-310-001), Zwick/Roell innovation project of college student fund, and Grant 10JC1411700 from the Science and Technology Commission of Shanghai Municipality.

\section{References}

[1] T. Lin and X. Wang, Encyclopedia of Nanoscience and Nanotechnology, edited by H. S. Nalwa, American Scientific, Los Angeles, Calif, USA, 2nd edition, 2011.

[2] L. Ji, Z. Lin, A. Medford, and X. Zhang, "In-situ encapsulation of nickel particles in electrospun carbon nanofibers and the resultant electrochemical performance," Chemistry $A$, vol. 15, no. 41, pp. 10718-10722, 2009.

[3] H. Niu, J. Zhang, Z. Xie, X. Wang, and T. Lin, "Preparation, structure and supercapacitance of bonded carbon nanofiber electrode materials," Carbon, vol. 49, no. 7, pp. 2380-2388, 2011.

[4] X. Wang, H. Niu, X. Wang, and T. Lin, "Needleless electrospinning of fine and uniform nanofibers using spiral coil spinnerets," Journal of Nanomaterials, vol. 2012, Article ID 785920, 9 pages, 2012.

[5] D. G. Yu, C. Branford-White, G. R. Williams et al., "Selfassembled liposomes from amphiphilic electrospun nanofibers," Soft Matter, vol. 7, pp. 8239-8246, 2011.

[6] D. G. Yu, C. Branford-White, S. W. A. Bligh, K. White, N. P. Chatterton, and L. M. Zhu, "Improving polymer nanofiber quality using a modified co-axial electrospinning process," Macromolecular Rapid Communications, vol. 32, no. 9-10, pp. 744-750, 2011.

[7] D. G. Yu, L. M. Zhu, C. Branford-White et al., "Solid dispersions in the form of electrospun core-sheath nanofibers," International Journal of Nanomedicine, vol. 6, pp. 3271-3280, 2011.

[8] D. G. Yu, P. Lu, C. Branford-White, J. H. Yang, and X. Wang, "Polyacrylonitrile nanofibers prepared using co-axial electrospinning with $\mathrm{LiCl}$ solution as sheath fluid," Nanotechnology, vol. 22, Article ID 435301, 7 pages, 2011.
[9] D. G. Yu, K. White, J. H. Yang, X. Wang, W. Qian, and Y. Li, "PVP nanofibers prepared using co-axial electrospinning with salt solution as sheath fluid," Materials Letter, vol. 67, pp. 7880, 2012.

[10] X. Lu, C. Wang, and Y. Wei, "One-dimensional composite nanomaterials: synthesis by electrospinning and their applications," Small, vol. 5, no. 21, pp. 2349-2370, 2009.

[11] Y. Dou, K. Lin, and J. Chang, "Polymer nanocomposites with controllable distribution and arrangement of inorganic nanocomponents," Nanoscale, vol. 3, no. 4, pp. 1508-1511, 2011.

[12] D. G. Yu, L. D. Gao, K. White, C. Branford-White, W. Y. $\mathrm{Lu}$, and L. M. Zhu, "Multicomponent amorphous nanofibers electrospun from hot aqueous solutions of a poorly soluble drug," Pharmaceutical Research, vol. 27, no. 11, pp. 2466-2477, 2010.

[13] L. Ji, Z. Lin, M. Alcoutlabi, and X. Zhang, "Recent developments in nanostructured anode materials for rechargeable lithium-ion batteries," Energy and Environmental Science, vol. 4, no. 8, pp. 2682-2699, 2011.

[14] Z. Dong, S. J. Kennedy, and Y. Wu, "Electrospinning materials for energy-related applications and devices," Journal of Power Sources, vol. 196, no. 11, pp. 4886-4904, 2011.

[15] X. Zhang, L. Ji, O. Toprakci, and M. Alcoutlabi, "Electrospun nanofiber-based anodes, cathodes, and separators for advanced lithium-ion batteries," Polymer Reviews, vol. 51, pp. 239-264, 2011.

[16] L. Ji, Z. Lin, B. Guo, A. J. Medford, and X. Zhang, "Assembly of carbon- $\mathrm{SnO}_{2}$ core-sheath composite nanofibers for superior lithium storage," Chemistry A, vol. 16, no. 38, pp. 11543$11548,2010$.

[17] C. Huang, S. J. Soenen, J. Rejman et al., "Stimuli-responsive electrospun fibers and their applications," Chemical Society Reviews, vol. 40, no. 5, pp. 2417-2434, 2011.

[18] X. Fang, H. Ma, S. Xiao et al., "Facile immobilization of gold nanoparticles into electrospun polyethyleneimine/polyvinyl alcohol nanofibers for catalytic applications," Journal of Materials Chemistry, vol. 21, no. 12, pp. 4493-4501, 2011.

[19] D. G. Yu, L. M. Zhu, S. W. A. Bligh, C. Branford-White, and K. White, "Coaxial electrospinning with organic solvent for controlling the size of self-assembled nanoparticles," Chemical Communications, vol. 47, no. 4, pp. 1216-1218, 2011.

[20] Anonymity, 2011, http://acupuncturetoday.com/herbcentral/ borneol.php.

[21] J. Pitha and T. Hoshino, "Effects of ethanol on formation of inclusion complexes of hydroxypropylcyclodextrins with testosterone or with methyl orange," International Journal of Pharmaceutics, vol. 80, no. 2-3, pp. 243-251, 1992.

[22] T. Loftsson and M. E. Brewster, "Pharmaceutical applications of cyclodextrins. 1. Drug solubilization and stabilization," Journal of Pharmaceutical Sciences, vol. 85, no. 10, pp. 10171025, 1996.

[23] D. G. Yu, N. P. Chatterton, J. H. Yang, X. Wang, and Y. Z. Liao, "Co-axial electrospinning with Triton X-100 solutions as sheath fluids for preparing PANnanofibers," Macromolecule and Materials Engineering. In press.

[24] J. M. Yang and D. G. Yu, "Co-axial electrospinning with sodium thiocyanate solution for preparing polyacrylonitrile nanofibers," Journal of Polymer Research, vol. 19, article 9789, 7 pages, 2011.

[25] D. G. Yu, G. R. Williams, L. D. Gao, S. W. A. Bligh, J. H. Yang, and X. Wang, "Polyacrylonitrile nanofibers prepared by coaxial electrospinning with a surfactant solution," Colloids and Surface Part A, vol. 396, pp. 161-168, 2012. 
[26] D. G. Yu, C. Branford-White, N. P. Chatterton et al., "Electrospinning of concentrated polymer solutions," Macromolecules, vol. 43, no. 24, pp. 10743-10746, 2010.

[27] X. X. Shen, D. G. Yu, L. M. Zhu, C. Branford-White, K. White, and N. P. Chatterton, "Electrospun diclofenac sodium loaded Eudragit L100-55 nanofibers for colon-targeted drug delivery," International Journal of Pharmaceutics, vol. 408, no. 1-2, pp. 200-207, 2011.

[28] D. G. Yu, X. X. Shen, C. Branford-White, K. White, L. M. Zhu, and S. W. A. Bligh, "Oral fast-dissolving drug delivery membranes prepared from electrospun polyvinylpyrrolidone ultrafine fibers," Nanotechnology, vol. 20, no. 5, Article ID 055104, 9 pages, 2009. 

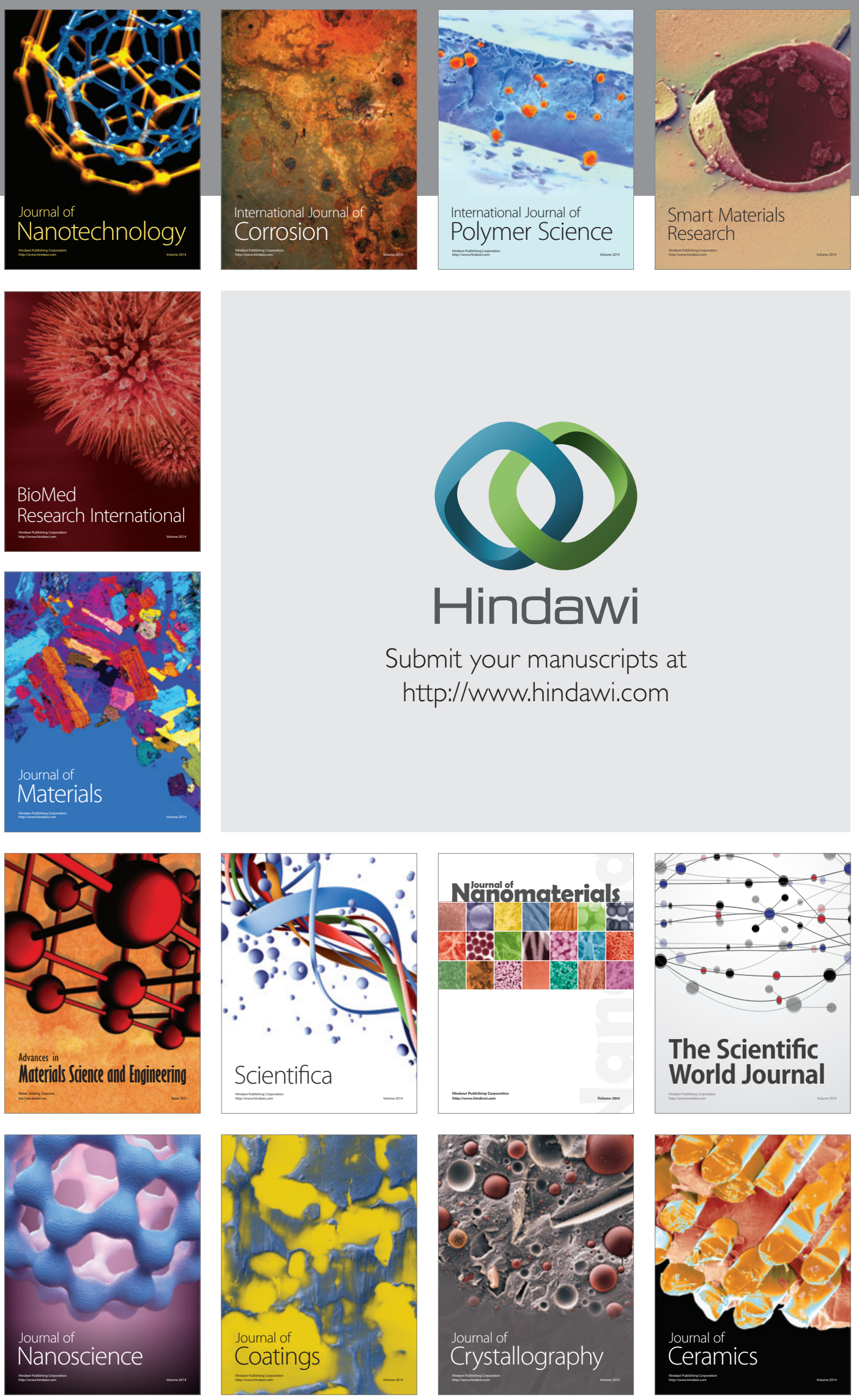

The Scientific World Journal

Submit your manuscripts at

http://www.hindawi.com

\section{World Journal}

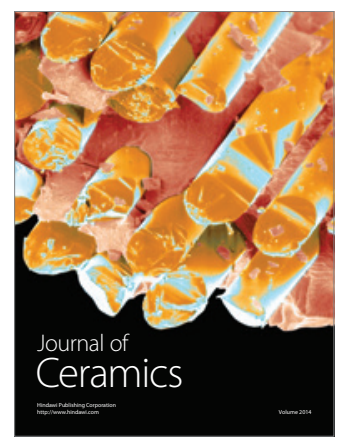

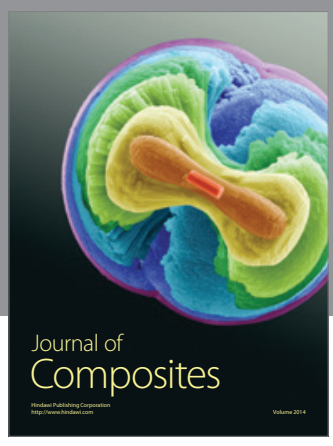
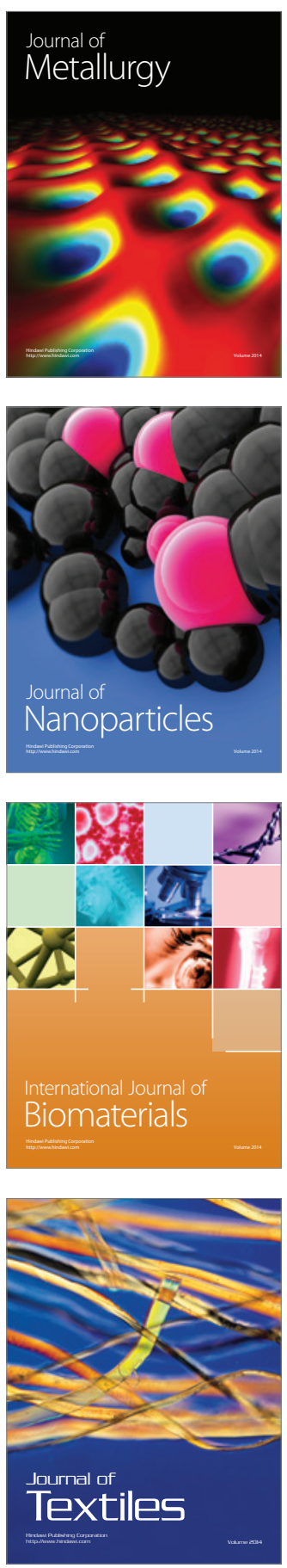